\title{
Effect of a Modified Pozzolan on the Sulphate Attack Resistant of Mortar
}

\author{
Bolanle D. Ikotun and Adeshina G. Ikotun
}

\begin{abstract}
Durability of mortar/concrete is a vital property that affects its serviceability. One of the factors that affect concrete durability is sulphate attack, which can result to expansion, cracking, deterioration, and deformation of concrete structures. The effect of a modified pozzolan (PWC) on the sulphate attack resistant of mortar was investigated in this study The additive, a blend of selected alkaloids and zeolite, is commercially available and effectively used in soil stabilization for road construction. The additive pozzolanic behaviour had been observed in previous study in terms of strength and permeability. However, the present study focuses on its resistant to sulphate attack as a pozzolan. PWC additive was used in the proportions of $0 \%, 0.4 \%, 0.6 \%$ and $2.5 \%$ by weight of cement. Samples were subjected to both internal and external sulphate attack tests, according to ASTM C1038 and ASTM C1012 respectively, after being cured for $14,28,60,90,120,180$, and 295 days. The results showed that PWC additive when used at low dosages of $0.4 \%$ and $0.6 \%$ PWC causes reduction in expansion due to external sulphate attack. At higher dosages of PWC additive, greater external expansion occurs when compared to control samples.
\end{abstract}

Index Terms-Durability, expansion, modified pozzolan, mortar, resistant, sulphate attack.

\section{INTRODUCTION}

Sulphate attack is one of the most aggressive environmental factors that affect long term durability of concrete structures. It can result in cracking, expansion and deterioration of concrete structures [1]. Sulphate attack is the reaction of sulphate ions with calcium hydroxide and calcium aluminate hydrate to form ettringite and gypsum, these products are voluminous and lead to expansion, cracking, deterioration, and deformation of concrete structures [1]-[4] when formed after concrete has hardened.

Sulphate attack can also lead to leaching of calcium compounds, degradation of calcium silicate hydrate (C-S-H), and overall deterioration of cement paste matrix [1]. One of the most severe conditions for durability of concrete is the sulphate or acid environment caused by industrial wastes or chemical residues at re-claimed grounds [5]. Deterioration of concrete as a result of sulphate attack can be in form of internal attack due to sulphate content of the cement, and external attack due to exposure of concrete to sulphate environment. Both forms of sulphate attack are manifested by expansion and cracking of concrete.

External sulphate attack may occurs due to penetration of sulphate in solution (for example ground water), which is in

Manuscript received April 12, 2013; revised July 10, 2013.

B. D. Ikotun is with the University of South Africa, South Africa (e-mail: ikotubd@unisa.ac.za, ayekot100@yahoo.com). return have contact with concrete. The reaction will change the composition and microstructure of the concrete to which it has contact. The effect of the change might result to external cracking, expansion or loss of bond between the cement paste and aggregate. The general effect of the changes will be loss of concrete strength.

Internal sulphate attack occurs when source of sulphate, for example, sulphate-rich aggregate or excess gypsum content is incorporated during concrete mix. The sulphate phases transformation of over-sulphated cement is from anhydrite to gypsum and ettringite, and then to thaumasite. This will finally result to concrete mush [6]. Delayed ettringite formation (DEF) is a form of ettringite that occurs in hardened concrete that has been cured at elevated temperature and contains excess sulphate, which reacts with calcium- and aluminium- containing phases of the cement paste and leads to increase in volume and expansion. Concrete composition, curing conditions and exposure conditions affect the potential/degree of DEF. Thaumasite form of sulphate attack requires adequate supply of sulphate and carbonate and it continues to form until the calcium silicate hydrate is completely decomposed. It is relatively unusual form of sulphate attack; it is associated with low temperatures and very wet environments [6]. The oxide compositions of ettringite and thaumasite as reported by Hooton [6] are shown below:

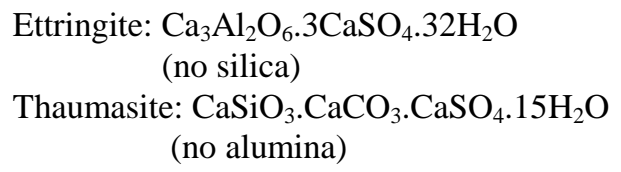

Quite a number of studies have been done to investigate ways of increasing concrete resistance to sulphate attack through incorporation of extenders (pozzolans) in mortars and concrete mixes [1], [2], [7], [8].

Consumption of calcium hydroxide produce during hydration by cement extenders and less presence of $\mathrm{C}_{3} \mathrm{~A}$ due to reduced quantity of cement content when extender is incorporated [4], [8], can help in increasing the resistance of concrete to sulphate attack. This emanates from reduction in gypsum and ettringite formation within the cementitious system. PWC additive has been investigated to be a pozzolanic reaction [9]; its effects on the sulphate resistant of mortar are explored in this paper.

\section{BRIEF REPORT FROM PREVIOUS WORK}

The previous investigation on the PWC additive as it affects concrete strength, permeability, sorptivity reported by 
Ikotun and Ekolu [9] and alkali silica reaction reported by Ikotun [10] are summarized below:

The use of PWC in concrete generally increases both early and late compressive strengths when compared to strength results of the control. At 28 days curing period, PWC concrete samples exhibited a decrease in split tensile strength compared to control, but at 180 days their split tensile strength increased to values higher than that of control samples. It was found that the effect of PWC additive on concrete strength improves significantly when it is used in conjunction with fly ash (FA). When $30 \%$ FA was used with $0.6 \%$ PWC additive in concrete, there was $21.3 \%$ increase in compressive strength at 180 days when compared to the control. With $0.6 \%$ PWC additive alone, a small increase of $7.5 \%$ in compressive strength was observed at 180 days. Accelerated compressive strength test done at 28 days on mortar samples also showed $22.7 \%$ increase in strength for $0.6 \%$ PWC $+30 \%$ FA compared to $12.6 \%$ strength increase for only $0.6 \%$ PWC additive. Oxygen permeability of concrete improved only when PWC additive was used in conjunction with FA. PWC additive improves concrete porosity and sorptivity but further effective reductions in the properties are observed when PWC is used together with FA. Results show that PWC additive is most effective when used in the presence of FA.

The use of PWC additive in reducing expansion due to alkali-silica reaction might be adverse. Higher expansions than for control were observed with PWC additive samples. This is explained by the very high alkali contents of PWC of $21 \%$ Na2Oe. When PWC additive was used together with FA in mixes, lower ASR expansions than for control were observed, this shows that PWC additive is most effective in reducing alkali silica reaction in the presence of FA.

\section{EXPERIMENTAL PROCEDURE}

\section{A. Materials}

The binding materials used for this study consisted of modified pozzolan (PWC) and ordinary Portland cement (OPC). PWC additive was commercially available and obtained from PowerCem Technologies, described as ConcreCem, micronized. The Portland cement used was the CEM 1 42.5N, produced by the Afrisam cement South Africa. Silica sand produced by Rolfes silica, South Africa was used as aggregate in mortar mixes. Silica sand was locally graded to conform to the requirement of SABS EN 196-1[11]; the locally graded silica sand (LGSS) was compared to the commercially available European standard silica sand (ESSS). The grading analysis of both LGSS and ESSS is shown in Fig. 1. Both LGSS and ESSS show similar trend, hence, LGSS is appropriate for the test. Calcium hydroxide $\left(\mathrm{Ca}(\mathrm{OH})_{2}\right)$ and sodium sulphate $\left(\mathrm{Na}_{2} \mathrm{SO}_{4}\right)$ were used as reagents.

\section{B. Sulphate Resistance}

According to ASTM C 1038 [12], expansion of samples due to their internal sulphate content was examined based on ASTM C 1038 [12]. The purpose of the test is to determine the amount of expansion of mortar bar samples when stored in water. PW WC additive was used as additive to OPC in the following proportions $0 \%, 0.4 \%, 0.6 \%$ and $2.5 \%$ by weight of cement. Details of all the mixes are shown in Table I.

According to ASTM C 1012 [13], expansion of samples when exposed to sulphate environment was examined based on ASTM C 1012 [13]. This test method provides a means of assessing the external sulphate resistance of mortars made using Portland cement, blends of Portland cement with pozzolans, and blended hydraulic cement. PWC additive was used as additive to OPC in the proportions of $0 \%, 0.4 \%$, $0.6 \%$ and $2.5 \%$ by weight of cement. Details of all the mixes are shown in Table II.

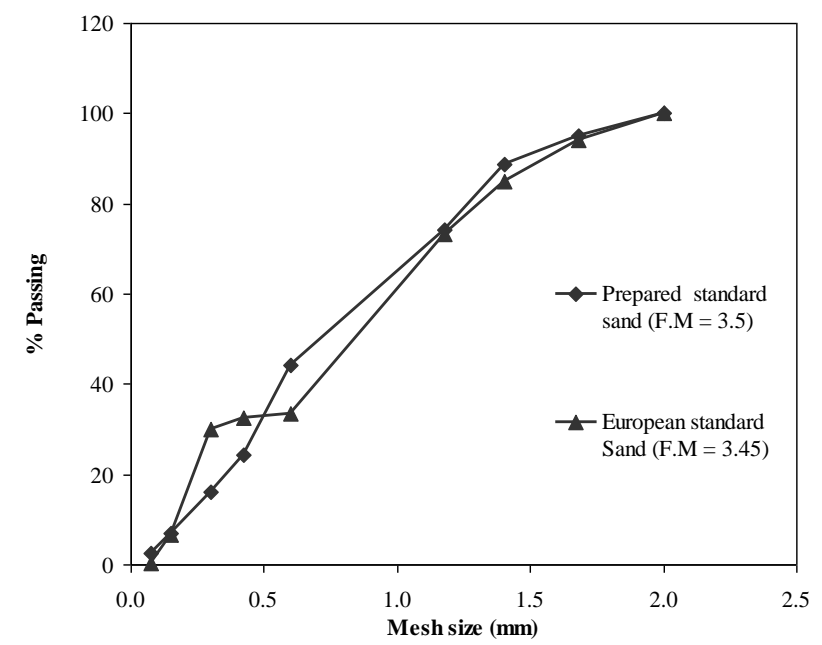

Fig.1. Grading curve of locally graded silica sand (prepared standard sand) and European standard sand.

\section{Moulds Preparation}

$25 \times 25 \times 280 \mathrm{~mm}$ prism moulds and $50 \mathrm{~mm}$ cubes (for external resistance test) were cleaned and sparingly covered with a lubricant, before mortar mixing operation. The lubricant was used as a releasing agent, so as to allow easy removal of hardened samples. The studs were attached at ends of $25 \times 25 \times 280 \mathrm{~mm}$ prisms moulds as shown in Fig. 2 .

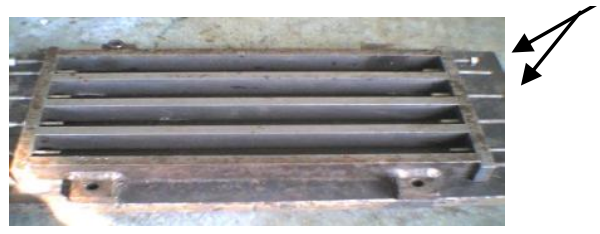

Fig. $2.25 \times 25 \times 280 \mathrm{~mm}$ prism mould with attached studs.

\section{Mixing Procedure}

OPC, PWC and locally graded silica sand of size $0.4-0.85$ mm (medium), were weigh batched and mixed in a HOBART mortar mixer for 3 minutes according to SABS EN 196-1[11]. Water and binder were mixed for 30 seconds at low speed, after which standard silica sand was then added over another 30 seconds. The mix was then allowed to stand for another one minute, while rubber scraper was used to remove the mortar adhering to the wall of the mixing bowl into the main mortar at the middle of the mixing bowl. The mixer was then adjusted to a medium speed and mixing run for another 1 minute. Constant water/binder (w/b) ratio of 0.485 was used as stipulated in ASTM C 1012 [13] (for external resistance test). 
TABLE I: MiXTURE Proportions FOR 1M3 OF MORTAR FOR SUlPHATES RESISTANCE Test TO C 1038 [12]

\begin{tabular}{|c|c|c|c|c|c|c|c|}
\hline \multicolumn{2}{|l|}{ Samples } & $\begin{array}{c}\text { Cement } \\
(\mathrm{Kg})\end{array}$ & $\begin{array}{c}\text { Silica sand } \\
(\mathrm{Kg})\end{array}$ & $\begin{array}{c}\text { PowerCem } \\
(\mathrm{Kg})\end{array}$ & $\begin{array}{c}\text { Water } \\
(\mathbf{K g})\end{array}$ & $\mathbf{w} / \mathbf{b}$ & $\begin{array}{l}\text { Flow } \\
(\mathbf{m m})\end{array}$ \\
\hline \multicolumn{2}{|l|}{ Control } & 714.00 & 1964.00 & 0.00 & 350.00 & 0.49 & 110.02 \\
\hline \multicolumn{2}{|c|}{$0.4 \%$ PWC + Cement } & 714.00 & 1964.00 & 2.86 & 351.00 & 0.49 & 110.04 \\
\hline \multicolumn{2}{|c|}{$0.6 \%$ PWC + Cement } & 714.00 & 1964.00 & 4.28 & 352.00 & 0.49 & 111.24 \\
\hline \multicolumn{2}{|c|}{$.5 \%$ PWC + Cement } & 714.00 & 1964.00 & 17.85 & 358.00 & 0.49 & 115.90 \\
\hline Samples & $\begin{array}{c}\text { Cement } \\
(\mathrm{Kg})\end{array}$ & $\begin{array}{l}\text { Silica sand } \\
\quad(\mathrm{Kg})\end{array}$ & $\begin{array}{c}\text { PowerCem } \\
\text { (Kg) }\end{array}$ & $\begin{array}{c}\text { Water } \\
\text { (Kg) }\end{array}$ & $\mathbf{w} / \mathbf{b}$ & \multicolumn{2}{|c|}{$\begin{array}{c}\text { Compressive } \\
\text { Strength }\left(\mathbf{N} / \mathbf{m m}^{2}\right) \\
\text { At day } 1\end{array}$} \\
\hline Control & 714.000 & 1964.000 & 0.000 & 346.300 & 0.485 & \multicolumn{2}{|c|}{24.200} \\
\hline $0.6 \% \mathrm{PWC}+$ Cement & 714.000 & 1964.000 & 4.280 & 348.400 & 0.485 & \multicolumn{2}{|c|}{22.800} \\
\hline $5 \%$ PWC + Cement & 714.000 & 1964.000 & 17.840 & 354.900 & 0.485 & \multicolumn{2}{|c|}{20.160} \\
\hline
\end{tabular}

\section{E. Casting and Compaction}

Mortar was cast by filling the already prepared $25 \times 25 \times$ $280 \mathrm{~mm}$ prisms moulds in two layers and compacting with tamping wooden plate of size $10 \times 25 \times 150 \mathrm{~mm}$. For external resistant test, $50 \mathrm{~mm}$ cube moulds were also filled and hand-held on a mechanical vibrating table for 10 seconds. Sufficient mortar was added to fill the $50 \mathrm{~mm}$ cubes mould, held on the vibrating table for further period of 10 seconds.

\section{F. Curing}

After casting, the moulds were covered with moist cloth for 24 hours to preserve the initial moisture condition of the sample, after which the hardened prisms were removed from their moulds and properly labeled. The samples were then placed in saturated lime (calcium hydroxide) solution for 30 minutes prior to making the initial measurement.

After the initial measurement, the samples were immersed in a saturated lime solution placed in a storage container. Bottom of the container was lined with plastic mesh to allow for easy expansion measurement. The container was covered tightly with a lid during sample storage. Periodically, the calcium hydroxide solution was re-filled to cater for the loss of solution which occurred as a result of evaporation or during removal of samples.

For external resistance test, immediately after molding, the filled mortar moulds were covered with a rigid plastic plate, and placed in a water curing tank. The temperature of curing water was maintained at $38 \pm 2{ }^{\circ} \mathrm{C}$ with a built in thermostat and a small circulation pump, until the mortar cube strength reached a value of $20 \mathrm{MPa}$. This strength value was reached after one day of curing for all cube samples tested. Initial measurement was then recorded, after which the bar samples were immersed in $5 \% \mathrm{Na}_{2} \mathrm{SO}_{4}$ solution in a storage plastic container. The samples rested on plastic mesh placed at the bottom of container to allow for free expansion movement.

The container was covered tightly with a lid during samples storage period. The storage container was periodically re-filled with $5 \% \mathrm{Na}_{2} \mathrm{SO}_{4}$ solution to maintain constant volume of solution.

\section{G. Testing}

Expansion was measured at different curing ages using length comparator as shown in Fig. 3. The initial measurement was recorded after one day of casting, while other expansions were measured at 14, 28, 60, 90, 120, 180, and 295 days curing period. For external resistance test, the initial measurement was recorded after compressive strength value of $20 \mathrm{MPa}$ has been confirmed. Other expansions were measured after $7,14,28,60,90,120$, and 180 days curing period.

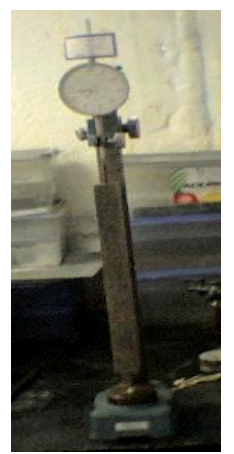

Fig. 3. Length comparator

\section{H. Expansion Calculation}

The change in length of the samples at any age was calculated as follows:

$$
\Delta L=\frac{L_{x}-L_{i}}{L_{g}} .100
$$

where:

$\Delta L=$ change in length at any age, $\%$

$L_{x}=$ comparator reading of samples at any age

$L_{i}=$ initial comparator reading of samples (reading at day 1)

$L_{g}=$ nominal gage length, $250 \mathrm{~mm}$

The change in length at any age was recorded as the expansion of the samples at that age. Results recorded are average of four samples.

\section{RESUlts AND DISCUSSION}

\section{A. Effect of PWC Additive on Internal Sulphate Attack of Mortar.}

Expansion of the samples when stored in lime water is directly related to the amount of sulphate in the cement according to ASTM C 1038 [12]. Fig. 4 shows the percentage expansion of samples due to sulphate attack when immersed 
in lime solution according to ASTM C 1038 [12]. There was an increase in expansion at the early days of curing between 14 and 28 days for control and $0.6 \%$ PWC samples, which might be as a result of incomplete hydration. Samples containing $0.6 \%$ PWC have highest expansion value of $0.033 \%$ at 28 days. Relatively moderate expansion was however observed for $0.6 \%$ PWC sample between 60 and 120 days. A similar expansion trend was also observed for all the samples between 120 and 295 days with higher expansion observed with samples containing PWC. This is an indication that the sulphate contents in blended samples (samples containing PWC) are higher than that of the control sample. This observation does not necessary explain the effect of the samples when exposed to sulphate environment. Internal Expansion may become excessive when the cement contains too much sulphate.

\section{B. Effect of PWC Additive on External Sulphate Attack of Mortar.}

Resistance of concrete to external sulphate environment is directly affected by how dense and permeable the concrete is. A dense and low permeable concrete will have good resistant to external sulphate environment than a porous and high permeable concrete. Fig. 5 shows the percentage expansion of samples due to sulphate attack when immersed in $5 \%$ $\mathrm{Na}_{2} \mathrm{SO}_{4}$ solution according to ASTM C 1012 [13]. At all ages observed, the expansion of $0.4 \%$ PWC and $0.6 \%$ PWC samples were less than the expansion observed in control samples. It was also observed that the higher dosage of $2.5 \%$ PWC additive resulted in a higher expansion. Expansion is seen to decrease for $0.4 \%$ PWC and $0.6 \%$ PWC samples at ages 28, 60, and 90 days. Further relative decrease in expansion was also observed for $0.4 \%$ PWC and $0.6 \%$ PWC samples at the late ages of 120,180 , and 260 days.

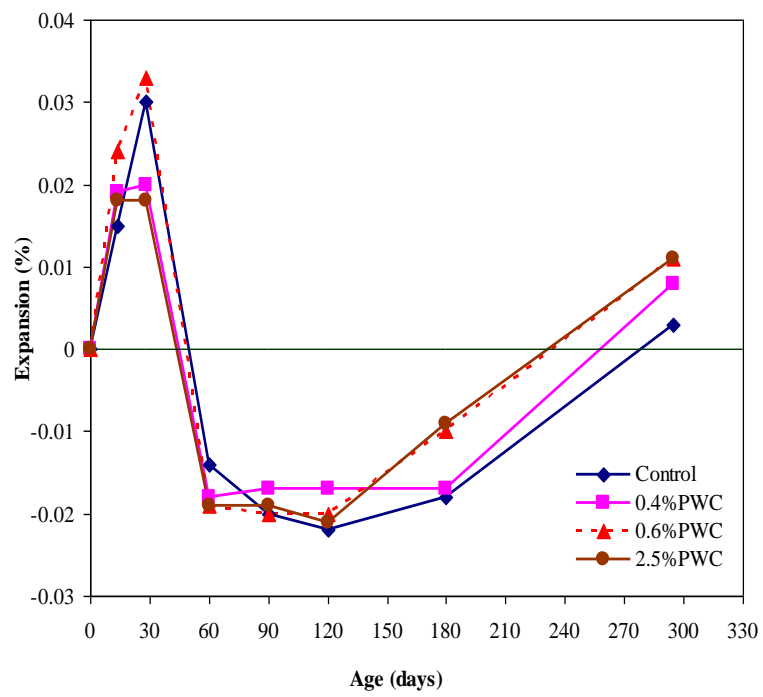

Fig. 4. Expansion due to internal sulphate attack as per ASTM C 1038 [12].

The influence of PWC additive on sulphate attack may partially be attributed to the pozzolanic reaction between the additive and $\mathrm{Ca}(\mathrm{OH})_{2}$ formed during hydration process. This reaction results into secondary $\mathrm{C}-\mathrm{S}-\mathrm{H}$ and forms more dense mortar and pores of similar diameter. Consumption of excess calcium hydroxide due to pozzolanic reaction is reported by Sideris et al. [3] to render it unavailable for the formation of ettringite and gypsum compounds. The formation of ettringite and gypsum compounds in hardened cementitious systems is responsible for expansion.

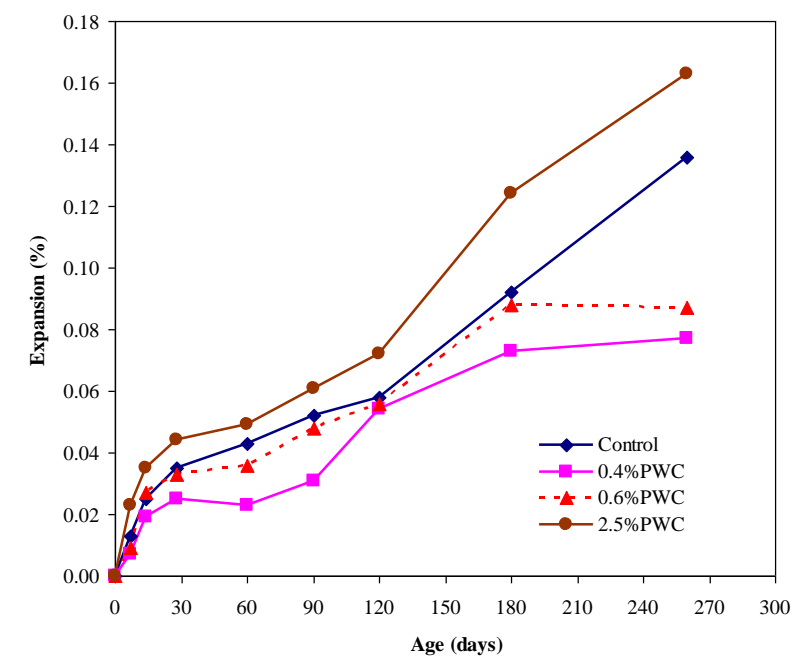

Fig. 5. Expansion due to external sulphate attack as per ASTM C 1012 [13].

\section{CONCLUSION}

It was found that when PWC additive is used at low dosages of $0.4 \%$ and $0.6 \%$ PWC, it causes reduction in expansion due to external sulphate attack. Though blended samples seems to have higher sulphate content, they exhibited better resistant to external sulphate attack when used optimally (0.4\% PWC and $0.6 \% \mathrm{PWC})$. At higher dosages of PWC additive, greater expansion occurs when compared to control samples. Again this result indicates that the optimum proportion of PWC additive to be used may lie in the range between $0.4 \%$ and $0.6 \%$.

\section{ACKNOWLEDGMENT}

The authors are grateful to:

- The PowerCem (Africa) Technologies, for providing a research grant to carry out this work.

- The University of the Witwatersrand, South Africa for providing the laboratory facilities.

- The University of South Africa for providing funding to attend the conference.

\section{REFERENCES}

[1] M. A. Nabil, "Durability of metakaolin concrete to sulfate attack," Cement and Concrete Research, vol. 36, pp. 1727-1734, September 2006.

[2] K. Torri, K. Taniguchi, and M. Kawamura, "Sulfate resistance of high ash content concrete," Cement and Concrete Research, vol. 25, pp. 759-768, May 1995.

[3] K. K. Sideris, A. E. Savva, and Papayianni "Sulfate resistance and carbonation of plain and blended cement," Cement and Concrete Composites, vol. 28, pp. 47-56, January 2006.

[4] U. A. Salah, "Sulfate resistance of plain and blended cements exposed to magnesium sulfate solutions," Construction and Building Materials, vol. 21, pp. 1792-1802, August 2007.

[5] B. Hanifi and A. Orhan, "Sulfate resistance of plain and blended cement," Cement and Concrete Composites, vol. 28, pp. 39-46, January 2006.

[6] R. D. Hooton. (CITED, April 2013). A review of different forms of sulfate attack. University of Toronto, Department of Civil Engineering. [Online]. Available: http://srnl.doe.gov/grout_present/2b_02_Hooton.pdf 
[7] G. J. Osborne, "Durability of Portland blast - furnace slag cement concrete," Cement and Concrete Composites, vol. 21, pp. 11-21, 1999.

[8] R. E. Rodriguez-Camacho and R. Uribe-Afif, "Important of using the natural pozzolans on concrete durability," Cement and Concrete Research, vol. 32, pp. 1851-1858, December 2002.

[1] B. D. Ikotun and S. Ekolu, "Strength and durability effect of modified zeolite additive on concrete properties," Construction and Building Materials, vol. 24, pp. 749-757, May 2010.

[9] B. D. Ikotun, "Effect of a modified zeolite additive on alkali silica reaction of mortar," in Construction: Essay on architectural history, theory and technology, N. Patricios and S. Alifragkis, Ed., Athens institute of education and research, ISBN 978-960-9549-89-9, 2012, pp 305-312.

[10] Methods of testing cement, Part 1: Determination of strength, SABS EN 196-1, pp. 4-14, 1994.

[11] Standard test method for expansion of hydraulic-cement mortar bars stored in water, ASTM C 1038, in Annual Book of ASTM Standards, vol. 04-01, pp. 517-519, 2006.

[12] Standard test method for length change of hydraulic-cement mortars exposed to a sulfate solution, ASTM C 1012, in Annual Book of ASTM Standards, vol. 04-01, pp. 509-514, 2006.

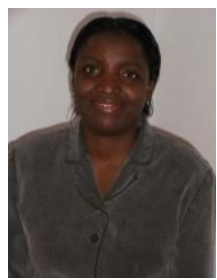

Bolanle Deborah Ikotun is a researcher and a senior lecturer in Civil Engineering department, University of South Africa. She has MSc (Eng) and B.Eng degrees in Civil Engineering from the University of the Witwatersrand, South Africa and The Federal University of Technology Akure, Nigeria respectively. She started her lecturing career in Nigeria, where she lectured for six years.
She later joined the University of South Africa as a contract staff in 2008 and the University of Johannesburg, South Africa as a temporary lecturer in 2009. She was given a permanent position as a lecturer in the University of South Africa in 2010. She has several published research papers in journals, conference proceedings and book chapter. She is currently pursuing her doctoral programme in the University of Johannesburg, South Africa in the department of Civil Engineering technology.

Mrs Ikotun research interests are in concrete mix design, concrete structural and durability properties testing, chemical deterioration of concrete, mechanical testing of concrete, concrete quality optimization techniques and investigation of extenders as they affect mortar and concrete properties. She is a member of Nigerian society of Engineer (NSE) and Concrete society of Southern Africa (CSSA).

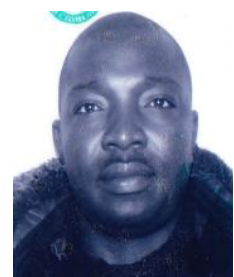

Adeshina Gabriel Ikotun is a postgraduate student in Chemical Engineering Department, University of Johannesburg, South Africa. He has B.Eng degree in Mining Engineering from the Federal University of Technology Akure, Nigeria. He worked as a mining Engineer at an Iron ore mining company in Nigeria for four years and as a senior mining Engineer for three years.

His curiosity on the optimisation of mineral recovery from mining products led him to pursuing his master's degree in Chemical Engineering at the University of Johannesburg, South Africa, focusing on the recovery of pentlandite in a low grade nickel sulphide ore. He has contributed to knowledge through journal articles and conference proceedings.

Mr Ikotun research interests are in the optimisation of mineral recovery from mining products and mineral processing. 